\title{
Super-Resolution Ultrasound Image Filtering with Machine-Learning to Reduce the Localization Error
}

\author{
Sevan Harput*,1,2, Long Hin Fong*, ${ }^{*}$, Antonio Stanziola ${ }^{1,3}$, Ge Zhang $^{1}$, Matthieu Toulemonde ${ }^{1}$, Jiaqi Zhu ${ }^{1}$, \\ Kirsten Christensen-Jeffries ${ }^{4}$, Jemma Brown ${ }^{4}$, Robert J. Eckersley ${ }^{4}$, \\ Enrico Grisan ${ }^{3,4}$, Chris Dunsby ${ }^{5}$, and Meng-Xing Tang ${ }^{1}$ \\ ${ }^{1}$ ULIS Group, Department of Bioengineering, Imperial College London, London, SW7 2BP, UK \\ ${ }^{2}$ Division of Electrical and Electronic Engineering, London South Bank University, London, SE1 0AA, UK \\ ${ }^{3}$ Department of Information Engineering, University of Padova, Padova, IT \\ ${ }^{4}$ Biomedical Engineering Department, Division of Imaging Sciences, King's College London, SE1 7EH, London, UK \\ ${ }^{5}$ Department of Physics and the Centre for Pathology, Imperial College London, London, SW7 2AZ, UK \\ ${ }^{*}$ These authors contributed equally to this work. \\ E-mail: S.Harput@1sbu.ac.uk, Mengxing.Tang@imperial.ac.uk
}

\begin{abstract}
Localization-based super-resolution imaging requires accurate detection of spatially isolated microbubbles. The reason for this requirement is that interfering or overlapping signals resulting from multiple microbubbles within the resolution limit can cause position errors. In addition to this, noise and artefacts (e.g. residual tissue signal after tissue-microbubble separation) further reduce the quality and hence the spatial resolution in SR imaging. Therefore, correctly identifying the echoes as noise, single microbubble, multiple microbubbles, or artefact is important.

In this study, the use of fast classification methods for identification and rejection of non-single microbubble echoes were demonstrated. Most commonly used supervised classification methods, including Decision Trees, Discriminant Analysis, Logistic Regression, Support Vector Machine, Ensembles, k-Nearest Neighbors, and Naive Bayes, were implemented for filtering artefacts and noise in super-resolution ultrasound images. Results showed that the Ensemble method, explicitly designed to deal with unbalanced data, achieved the best result since most of the localized events are true microbubbles, which is typical for super-resolution imaging datasets.
\end{abstract}

\section{INTRODUCTION}

Localization-based super-resolution ultrasound (SR-US), also known as ultrasound localization microscopy, relies on detection of microbubbles positions with high precision. Although this method is adapted from optical super-resolution microscopy, many studies have contributed to the development of localization-based SR-US imaging using microbubbles [1] [17] and nanodroplets [18]-[21]. These developments are explained in detail by a recent review [22].

SR-US imaging can potentially achieve a localization precision, which determines the image resolution, of a few micrometers at medical ultrasound imaging frequencies (1$10 \mathrm{MHz}$ ) [1], [5]. However, localization precision and image quality might be reduced due to many reasons in practice. Since multiple overlapping microbubble echoes within the resolution limit can cause position errors, isolated microbubble echoes should be localized with the best possible method [23], [24]. Super-resolved images are generated by combining the locations of spatially isolated microbubbles through multiple frames and hence motion can significantly reduce the image quality and increase the localization error. If motion estimation and correction are applied to improve the superresolution image, then motion correction accuracy will limit the achievable spatial resolution [10], [25], [26]. In addition, noise and tissue artefacts in the contras-mode image further reduce the quality and spatial resolution. Therefore, correctly identifying the echoes as noise, single microbubble, multiple microbubbles, or artefact is crucial. In this study, we use a set of supervised classification methods for identification and rejection of non-single microbubble echoes.

\section{Materials AND Methods}

\section{A. Experimental Setup}

The ULA-OP system (MSD Lab, University of Florence, Italy) was used to acquire data using the LA332 ultrasound probe (Esaote, Firenze, Italy) [27], [28]. This 144 element linear probe had a $-6 \mathrm{~dB}$ bandwidth ranging from $2-7.5 \mathrm{MHz}$ and an element pitch of $254 \mu \mathrm{m}$.

Super-resolution filtering was tested on an in vitro experimental setup with a $200 \mu \mathrm{m}$ cellulose tube fixed in paraffin gel as shown in the Figure 1 (left). A 1:6000 times diluted Sonovue (Bracco S.p.A, Milan, Italy) solution was flown through the cellulose tube during the measurements. Plane wave imaging was performed at a center frequency of $4 \mathrm{MHz}$. A total of 1000 frames were acquired with a pulse repetition frequency of $100 \mathrm{~Hz}$. All frames were filtered by using singular value decomposition to remove the tissue echoes and generate a contrast-mode image [29].

\section{B. Super-resolution using Statistical Classification}

Super-localization was performed on each ultrasound image after setting an image pixel value threshold to reject the noise and detect potential microbubble signals. The area, intensity, eccentricity, and solidity of each detected event was computed. 

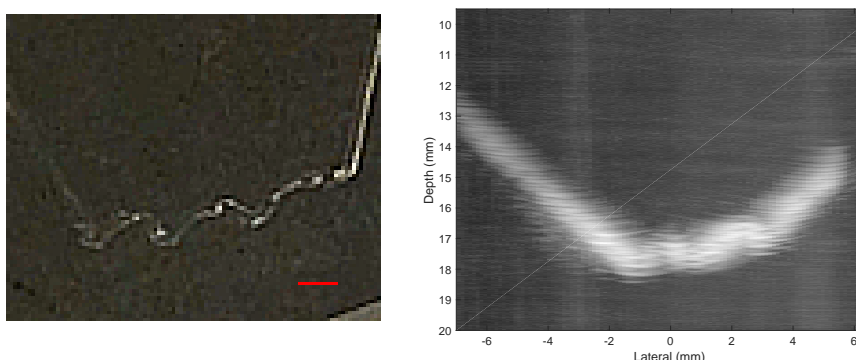

Fig. 1. (Left) Optical image of in vitro vessel phantom with a $200 \mu \mathrm{m}$ cellulose tube fixed in paraffin gel. A $1 \mathrm{~mm}$ red line is plotted on the image to show the scale. (Right) Maximum intensity projection of 1000 frames used to generate the super-resolution image with a dynamic range of $40 \mathrm{~dB}$
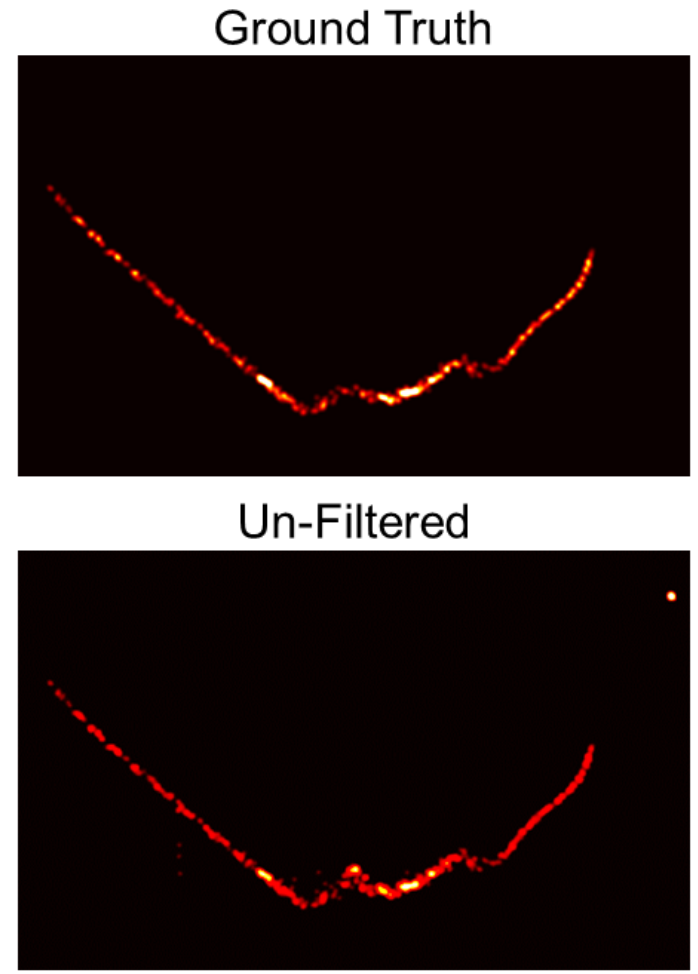

Fig. 2. (Top) The ground truth super-resolution image generated from manually labelled microbubbles by an expert user with the priori knowledge of the tube. (Bottom) Un-filtered super-resolution image generated by using every localization event.

All events were manually labelled as single microbubble or not, to provide the ground truth as shown in Figure 2 (top).

These shape parameters were used to filter the superresolution images by discarding multiple microbubbles, noise and artefacts in two ways: (1) Detected events were filtered by setting thresholds on each shape parameter separately; (2) Supervised learning approaches were implemented for filtering by implementing the following classification methods: Decision Trees, Discriminant Analysis (linear and quadratic), Logistic Regression, Support Vector Machine (with linear, quadratic and cubic kernels), Ensembles (bagged and boosted trees), k-Nearest Neighbors algorithm, and Naive Bayes. These learning algorithms were implemented in Matlab (The Math-
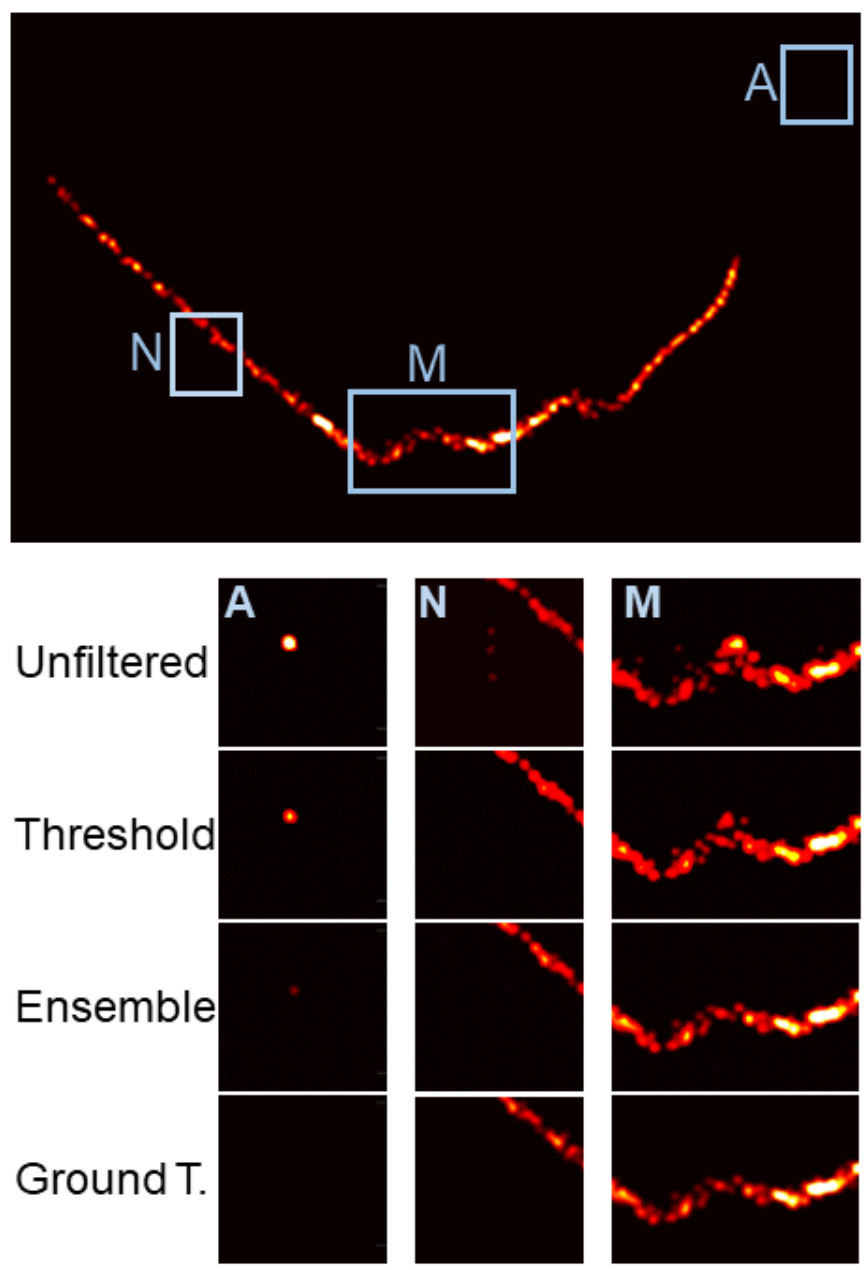

Fig. 3. (Top) Super-resolution image highlighting the region of interests for filtering with an artefact $[\mathrm{A}]$, noisy region $[\mathrm{N}]$, and region with multiple microbubbles $[\mathrm{M}]$. (Bottom) Comparison of the super-resolution images filtered by different methods at the region with artefact, noise, and multiple bubbles.

Works, Inc., Natick, MA) using their Statistics and Machine Learning Toolbox. The data set was manually labelled and they were trained and evaluated by 5 fold cross-validation.

\section{RESUlts \& Discussion}

Results are compared qualitatively in Figure 3 and quantitatively in Figure 4. The unfiltered super-resolution image achieved the highest sensitivity value of $100 \%$ (number of correctly classified microbubbles / true microbubbles). However, it only achieved a precision, which is more important for super-resolution imaging, value of $78 \%$ (number of correctly classified microbubbles / all predicted microbubbles). The thresholding method did not score over $93 \%$ in any test. The ensemble classification method using RUSboost decision tree as base classifier outperformed all methods with a precision of $96 \%$ and a sensitivity of $90 \%$.

The second best method, cubic SVM, achieved a slightly higher sensitivity of $92 \%$ while reducing the precision down to $94 \%$. The decent performance of cubic and quadratic SVM 


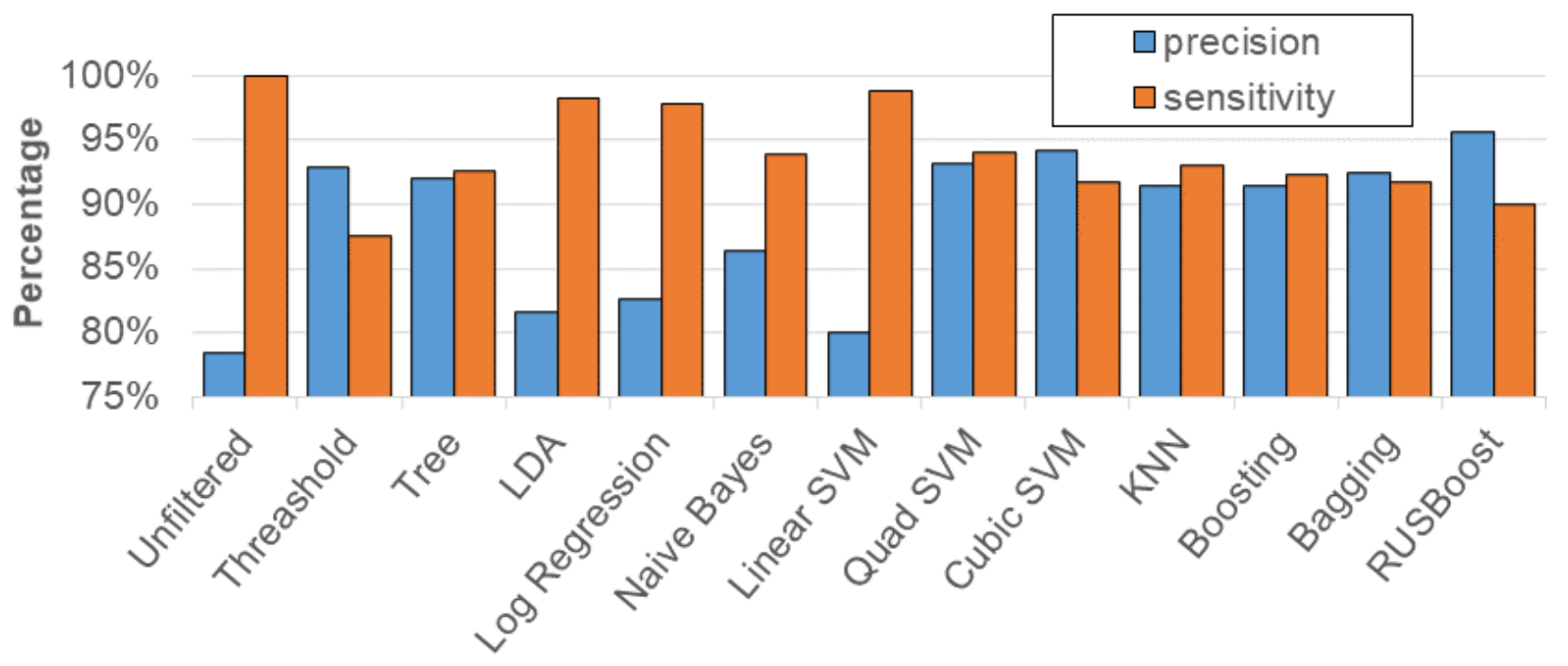

Fig. 4. Quantitative comparison of Precision (number of correctly classified microbubbles / all predicted microbubbles) and Sensitivity (number of correctly classified microbubbles/ number of true microbubbles) values for unfiltered, filtered with threshold (optimized for precision) and all tested statistical-classification methods.

suggest the four measured features (area, intensity, eccentricity and solidity) do a fair job separating the single microbubbles in the hyperspace.

The experiments in this study and most well-designed superresolution imaging experiments will have a skewed data distribution where most of the localized events are true microbubble signals. Among the all tested classification methods RUSBoost Tree, which is a good algorithm to train skewed data (number of true microbubbles $>>$ number of false microbubbles). The reason behind the suitability of this specific method for filtering super-resolution ultrasound image datasets can be explained with the theory of decision trees and ensembles.

Decision Tree Ensembles train several trees and make a combine the outputs for a final categorization. Bagging selects with replacement several random sets of observations and trains a tree on each set. New observations are then categorized by a vote of the collection of trees or the forest. Boosting selects with replacement a set of observations and trains a tree. It then trains the next tree on but weights the random selection to include more observations that have been incorrectly selected by the previous. Subsequent trees are generated in the same manner until the desired number of trees is reached. New observations are voted on with more weight given to accurate trees. Training 30 trees, both bagged and boosted forests had precisions of $92 \%$ and both had sensitivities of 92\%. A randomly under sampled boost (RUSBoost) ensemble was also implemented and it produced the highest precision with $96 \%$ and a sensitivity of $90 \%$. This variant produces the first random by under-sampling the larger class of observations to produce a training set with equal numbers of observations in each class. This technique has been known to improve results of unbalanced data sets such as ours where $78 \%$ of the observations are microbubbles.

\section{CONCLUSION}

The experiments in this study have a skewed data distribution where most of the localized events are true microbubbles, which is typical for super-resolution imaging datasets. The ensemble method (RUSBoost Tree), explicitly designed to deal with unbalanced data, achieved the best result. In the future, more unified and automated approaches to define the superresolution filtering criteria will be investigated.

Super-resolution image filtering with automated and fast methods is significant for the translation of this technology. The proposed filtering method can also be used to reduce the localization error in non-localization-based super-resolution ultrasound imaging methods that are aiming to reduce the acquisition time [30]-[32].

In conclusion, statistical classification is a promising filtering tool for super-resolution imaging with possibility of fast implementation using machine learning.

\section{ACKNOWLEDGMENTS}

This work was supported mainly by the EPSRC under Grant EP/N015487/1 and EP/N014855/1, in part by the King's College London (KCL) and Imperial College London EPSRC Centre for Doctoral Training in Medical Imaging (EP/L015226/1), in part by the Wellcome EPSRC Centre for Medical Engineering at KCL (WT 203148/Z/16/Z), in part by the Department of Health through the National Institute for Health Research comprehensive Biomedical Research Center Award to Guy's and St Thomas' NHS Foundation Trust in partnership with KCL and King's College Hospital NHS Foundation Trust, in part by the Graham-Dixon Foundation and in part by NVIDIA GPU grant. 


\section{REFERENCES}

[1] O. M. Viessmann, R. J. Eckersley, K. Christensen-Jeffries, M.-X. Tang, and C. Dunsby, "Acoustic super-resolution with ultrasound and microbubbles," Phys. Med. Biol, vol. 58, pp. 6447-6458, 2013.

[2] M. A. O'Reilly and K. Hynynen, "A super-resolution ultrasound method for brain vascular mapping," Medical Physics, vol. 40, no. 110701, 2013.

[3] Y. Desailly, O. Couture, M. Fink, and M. Tanter, "Sono-activated ultrasound localization microscopy," Applied Physics Letters, vol. 103, no. 174107,2013

[4] K. Christensen-Jeffries, R. J. Browning, M.-X. Tang, C. Dunsby, and R. J. Eckersley, "In vivo acoustic super-resolution and super-resolved velocity mapping using microbubbles," IEEE Trans. Med. Imag., vol. 34, no. 2, pp. 433-440, 2015.

[5] Y. Desailly, J. Pierre, O. Couture, and M. Tanter, "Resolution limits of ultrafast ultrasound localization microscopy," Phys. Med. Biol, vol. 60, pp. 8723-8740, 2015.

[6] C. Errico, J. Pierre, S. Pezet, Y. Desailly, Z. Lenkei, O. Couture, and M. Tanter, "Ultrafast ultrasound localization microscopy for deep superresolution vascular imaging," Nature, vol. 527, pp. 499-507, 2015.

[7] D. Ackermann and G. Schmitz, "Detection and tracking of multiple microbubbles in ultrasound b-mode images," IEEE Trans. Ultrason., Ferroelectr., Freq. Control, vol. 63, no. 1, pp. 72-82, 2016.

[8] J. Foiret, H. Zhang, T. Ilovitsh, L. Mahakian, S. Tam, and K. W. Ferrara, "Ultrasound localization microscopy to image and assess microvasculature in a rat kidney," Scientific Reports, vol. 7, no. 13662, pp. 1-12, 2017.

[9] F. Lin, S. E. Shelton, D. Espindola, J. D. Rojas, G. Pinton, and P. A. Dayton, "3-d ultrasound localization microscopy for identifying microvascular morphology features of tumor angiogenesis at a resolution beyond the diffraction limit of conventional ultrasound," Theranostics, vol. 7, no. 1, pp. 196-204, 2017.

[10] S. Harput, K. Christensen-Jeffries, J. Brown, Y. Li, K. J. Williams, A. H. Davies, R. J. Eckersley, C. Dunsby, and M. Tang, "Two-stage motion correction for super-resolution ultrasound imaging in human lower limb," IEEE Trans. Ultrason., Ferroelectr., Freq. Control, vol. 65, no. 5, pp. 803-814, 2018.

[11] P. Song, J. D. Trzasko, A. Manduca, R. Huang, R. Kadirvel, D. F. Kallmes, and S. Chen, "Improved super-resolution ultrasound microvessel imaging with spatiotemporal nonlocal means filtering and bipartite graph-based microbubble tracking," IEEE Trans. Ultrason., Ferroelectr., Freq. Control, vol. 65, no. 2, pp. 149-167, 2018.

[12] T. Opacic, S. Dencks, B. Theek, M. Piepenbrock, D. Ackermann, A. Rix, T. Lammers, E. Stickeler, S. Delorme, G. Schmitz, and F. Kiessling, "Motion model ultrasound localization microscopy for preclinical and clinical multiparametric tumor characterization," Nature Communications, vol. 9, no. 1527, pp. 1-13, 2018.

[13] T. Ilovitsh, A. Ilovitsh, J. Foiret, B. Z. Fite, and K. W. Ferrara, "Acoustical structured illumination for super-resolution ultrasound imaging," Communications Biology, vol. 1, no. 3, 2018.

[14] S. Harput, K. Christensen-Jeffries, J. Brown, J. Zhu, G. Zhang, C. H. Leow, M. Toulemonde, A. Ramalli, E. Boni, P. Tortoli, R. J. Eckersley, C. Dunsby, and M.-X. Tang, "3-d super-resolution ultrasound imaging using a 2-d sparse array with high volumetric imaging rate," in IEEE International Ultrasonics Symposium (IUS), 2018, pp. 1-4.

[15] J. Zhu, E. M. Rowland, S. Harput, K. Riemer, C. H. Leow, B. Clark, K. Cox, A. Lim, K. Christensen-Jeffries, G. Zhang, J. Brown, C. Dunsby, R. J. Eckersley, P. D. Weinberg, and M.-X. Tang, "3d super-resolution us imaging of rabbit lymph node vasculature in vivo by using microbubbles," Radiology, vol. 291, no. 3, pp. 642-650, 2019, pMID: 30990382.

[16] B. Heiles, M. Correia, V. Hingot, M. Pernot, J. Provost, M. Tanter, and O. Couture, "Ultrafast 3d ultrasound localization microscopy using a 3232 matrix array," IEEE Trans. Med. Imag., vol. 38, no. 9, pp. 20052015, 2019.

[17] S. Harput, K. Christensen-Jeffries, A. Ramalli, J. Brown, J. Zhu, G. Zhang, C. H. Leow, M. Toulemonde, E. Boni, P. Tortoli, R. J. Eckersley, C. Dunsby, and M.-X. Tang, "3-d super-resolution ultrasound imaging with a 2-d sparse array," IEEE Trans. Ultrason., Ferroelectr., Freq. Control, in press.
[18] G. P. Luke, A. S. Hannah, and S. Y. Emelianov, "Super-resolution ultrasound imaging in vivo with transient laser-activated nanodroplets," Nano Letters, vol. 16, pp. 2556-2559, 2016.

[19] H. Yoon, K. A. Hallam, C. Yoon, and S. Y. Emelianov, "Super-resolution imaging with ultrafast ultrasound imaging of optically triggered perfluorohexane nanodroplets," IEEE Trans. Ultrason., Ferroelectr., Freq. Control, vol. 65, no. 12, pp. 2277-2285, 2018.

[20] G. Zhang, S. Harput, S. Lin, K. Christensen-Jeffries, C. H. Leow, J. Brown, C. Dunsby, R. J. Eckersley, and M.-X. Tang, "Acoustic wave sparsely activated localization microscopy (awsalm): Super-resolution ultrasound imaging using acoustic activation and deactivation of nanodroplets," Applied Physics Letters, vol. 113, no. 1, p. 014101, 2018.

[21] G. Zhang, S. Harput, H. Hu, K. Christensen-Jeffries, J. Zhu, J. Brown, C. H. Leow, R. J. Eckersley, C. Dunsby, and M.-X. Tang, "Fast acoustic wave sparsely activated localization microscopy (fast-awsalm): Ultrasound super-resolution using plane-wave activation of nanodroplets," IEEE Trans. Ultrason., Ferroelectr., Freq. Control, vol. 66, no. 6, pp. 1039-1046, 2019.

[22] O. Couture, V. Hingot, B. Heiles, P. Muleki-Seya, and M. Tanter, "Ultrasound localization microscopy and super-resolution: A state of the art," IEEE Trans. Ultrason., Ferroelectr., Freq. Control, vol. 65, no. 8, pp. 1304-1320, 2018.

[23] K. Christensen-Jeffries, S. Harput, J. Brown, P. N. T. Wells, P. Aljabar, C. Dunsby, M.-X. Tang, and R. J. Eckersley, "Microbubble axial localization errors in ultrasonic super-resolution imaging," IEEE Trans. Ultrason., Ferroelectr., Freq. Control, vol. 64, no. 11, pp. 1644-1654, 2017.

[24] S. Harput, K. Christensen-Jeffries, J. Brown, R. J. Eckersley, C. Dunsby, and M.-X. Tang, "Localisation of multiple non-isolated microbubbles with frequency decomposition in super-resolution imaging," in IEEE International Ultrasonics Symposium (IUS), 2017, pp. 1-4.

[25] S. Harput, K. Christensen-Jeffries, Y. Li, J. Brown, R. J. Eckersley, C. Dunsby, and M.-X. Tang, "Two stage sub-wavelength motion correction in human microvasculature for ceus imaging," in IEEE International Ultrasonics Symposium (IUS), 2017, pp. 1-4.

[26] S. Harput, K. Christensen-Jeffries, J. Brown, J. Zhu, G. Zhang, R. J. Eckersley, C. Dunsby, and M.-X. Tang, "3-d motion correction for volumetric super-resolution ultrasound imaging," in IEEE International Ultrasonics Symposium (IUS), 2018, pp. 1-4.

[27] E. Boni, L. Bassi, A. Dallai, F. Guidi, V. Meacci, A. Ramalli, S. Ricci, and P. Tortoli, "Ula-op 256: A 256-channel open scanner for development and real-time implementation of new ultrasound methods," IEEE Trans. Ultrason., Ferroelectr., Freq. Control, vol. 63, no. 10, pp. 14881495, 2016.

[28] E. Boni, L. Bassi, A. Dallai, V. Meacci, A. Ramalli, M. Scaringella, F. Guidi, S. Ricci, and P. Tortoli, "Architecture of an ultrasound system for continuous real-time high frame rate imaging," IEEE Trans. Ultrason., Ferroelectr., Freq. Control, vol. 64, no. 9, pp. 1276-1284, 2017.

[29] C. Demene, T. Deffieux, M. Pernot, B.-F. Osmanski, V. Biran, J.-L. Gennisson, L.-A. Sieu, A. Bergel, S. Franqui, J.-M. Correas, I. Cohen, O. Baud, and M. Tanter, "Spatiotemporal clutter filtering of ultrafast ultrasound data highly increases doppler and fultrasound sensitivity," IEEE Trans Med Imaging, vol. 34, no. 11, pp. 2271-2285, 2015.

[30] A. Bar-Zion, C. Tremblay-Darveau, O. Solomon, D. Adam, and Y. C. Eldar, "Fast vascular ultrasound imaging with enhanced spatial resolution and background rejection," IEEE Trans. Med. Imag., vol. 36, pp. 169-180, 2017.

[31] R. J. van Sloun, O. Solomon, Y. C. Eldar, H. Wijkstra, and M. Mischi, "Sparsity-driven super-resolution in clinical contrast-enhanced ultrasound," in IEEE International Ultrasonics Symposium (IUS), 2017, pp. $1-4$.

[32] A. Bar-Zion, O. Solomon, C. Tremblay-Darveau, D. Adam, and Y. C. Eldar, "Sushi: Sparsity-based ultrasound super-resolution hemodynamic imaging," IEEE Trans. Ultrason., Ferroelectr., Freq. Control, vol. 65, no. 12 , pp. 2365-2380, 2018. 